2005-01-01

\title{
Study of Transmission Response of Edge Filters Employed in Wavelength Measurements
}

\author{
Qian Wang \\ Technological University Dublin \\ Gerald Farrell \\ Technological University Dublin, gerald.farrell@tudublin.ie \\ Thomas Freir \\ Technological University Dublin, thomas.freir@tudublin.ie
}

Follow this and additional works at: https://arrow.tudublin.ie/engscheceart

Part of the Electrical and Computer Engineering Commons

\section{Recommended Citation \\ Wang, Q, Farrell, G., Freir, T.:Study of Transmission Response of Edge Filters Employed in Wavelength Measurements.Applied Optics, Vol.44, no.36, p. 7789. doi:10.1364/A0.44.007789}

This Article is brought to you for free and open access by the School of Electrical and Electronic Engineering at ARROW@TU Dublin. It has been accepted for inclusion in Articles by an authorized administrator of ARROW@TU Dublin. For more information, please contact arrow.admin@tudublin.ie, aisling.coyne@tudublin.ie, gerard.connolly@tudublin.ie.

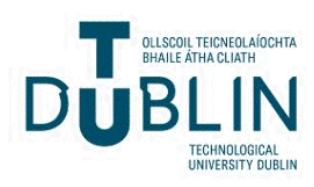




\title{
Study of transmission response of edge filters employed in wavelength measurements
}

\author{
Qian Wang, Gerald Farrell, and Thomas Freir
}

\begin{abstract}
The ratiometric wavelength-measurement system is modeled and an optimal design of transmission response of the employed edge filter is demonstrated in the context of a limited signal-to-noise ratio of the signal source. The corresponding experimental investigation is presented. The impact of the limited signal-to-noise ratio of the signal source on determining the optimal transmission response of edge filters for a wavelength-measurement application is shown theoretically and experimentally. (C) 2005 Optical Society of America
\end{abstract}

OCIS codes: $120.2440,120.4570,060.2380$.

\section{Introduction}

Wavelength measurements are required for many optical systems. Examples include wavelength measurement in a multichannel dense wavelength-divisionmultiplexing optical communication system and fiberBragg-grating-based optical sensing systems. ${ }^{1-5}$ There are different wavelength-measurement schemes, and among them the ratiometric detection scheme, ${ }^{2-5}$ which converts the wavelength measurement into a signal-intensity measurement, has a simple configuration and offers a high-speed measurement as compared with, e.g., wavelength-scanning-based active measurement schemes. The ratiometric detection scheme employs an edge filter and utilizes the transition region of its transmission response. The employed edge filters could be bulk thin-film filters, ${ }^{2}$ biconical fiber filters, ${ }^{4}$ fiber gratings, ${ }^{5}$ multimode interference couplers, ${ }^{6}$ directional couplers, ${ }^{7}$ and so on.

Previous publications about the ratiometric detection scheme have mainly focused on different types of edge filters, and there have been few investigations concerning the transmission response of the edge filter. Defining the transmission response, specifically the wavelength range and slope of the transition region, is the preliminary work in optimal design of these edge filters. A straightforward approach is to

The authors are with the Applied Optoelectronics Centre, School of Electronics and Communications Engineering, Dublin Institute of Technology, Kevin Street, Dublin 8, Ireland.

Received 8 July 2005; revised 18 August 2005; accepted 19 August 2005 .

0003-6935/05/367789-04\$15.00/0

(C) 2005 Optical Society of America make the slope of the transmission response for the edge filters as large as possible to ensure a high resolution of the measurement system, given the limited precision of power detectors. This paper studies the optimal transmission response of the edge filter for wavelength measurement in the context of the effect of a limited signal-to-noise ratio (SNR) of the signal source, e.g., signal to spontaneous-emission ratio for the lasers, which has not been addressed in related literature. In Section 2, the wavelengthmeasurement system involving the source, the edge filter, and the photodiodes is modeled and design of the transmission response of edge filters is subsequently demonstrated. Corresponding experimental investigations are presented in Section 3. Theoretical and experimental results indicate that the limited SNR of a signal source has a significant impact in the optimal design of the transmission response for the edge filters applied in wavelength measurements.

\section{Theoretical Modeling of Wavelength-Measurement Systems and Optimal Design of Edge Filter's Transmission Response}

Figure 1 gives the schematic configuration of a ratiometric wavelength-measurement system employing an edge filter. The input signal is split into two equal signals. One passes through a reference arm (arm B) and the other passes through the edge filter (arm A). Two photodiodes are placed at the ends of both arms. By measuring the ratio of the electrical outputs of the two photodiodes, we can determine the wavelength of the input signal assuming a suitable calibration has taken place.

In a ratiometric wavelength-measurement system, 


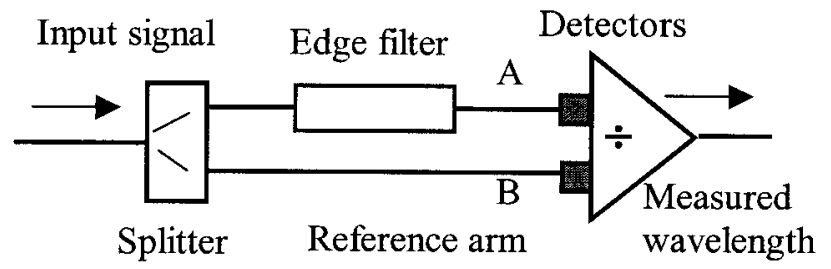

Fig. 1. Schematic configuration of edge-filter-based ratiometric wavelength-measurement system.

the narrowband input signal with a center wavelength $\lambda_{0}$ could be from a tunable laser or the reflection of a Bragg grating sensor. Such an input signal can be approximated by a Gaussian function with a spectral width $\Delta \lambda$ and center wavelength $\lambda_{0}$. In practice, the input signal generally has a limited SNR, which means that there is measurable power even far from the center wavelength in the spectrum. Figure 2 presents typical spectral distributions of the output intensity from a tunable laser at different central wavelengths, which are measured by an optical spectral analyzer in a wavelength range from 1500 to $1600 \mathrm{~nm}$. From these measured spectral distributions one can see that, for this tunable laser, the spontaneous-emission ratio is $>40 \mathrm{~dB}$ and it has different values for different output center wavelengths. Therefore, considering the SNR the output spectral response of source can be simply described $a^{2,8,9}$ (suppose the power at the peak wavelength is $0 \mathrm{dBm}$ )

$$
\begin{aligned}
& 10 \log _{10}\left[I_{\lambda_{0}}(\lambda)\right]= \\
& \begin{cases}10 \log _{10}\left\{\exp \left[-4 \ln 2 \frac{\left(\lambda-\lambda_{0}\right)^{2}}{\Delta \lambda_{0}{ }^{2}}\right]\right\}, & \left|\lambda-\lambda_{0}\right| \leq \Omega, \\
-S+r\left(\lambda_{0}\right), & \left|\lambda-\lambda_{0}\right|>\Omega\end{cases}
\end{aligned}
$$

where $S$ is the SNR for the source, $r\left(\lambda_{0}\right)$ is a small random number (typical range is from 0 to $1 \mathrm{~dB}$ ), and $\Omega$ is determined by the source with a given SNR,

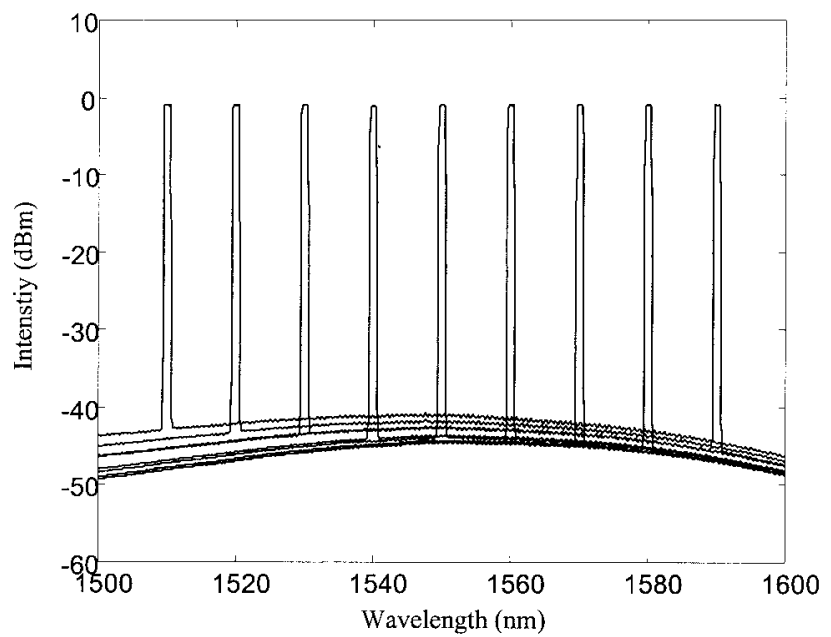

Fig. 2. Measured intensity distributions for a tunable laser in the wavelength range from 1500 to $1600 \mathrm{~nm}$.

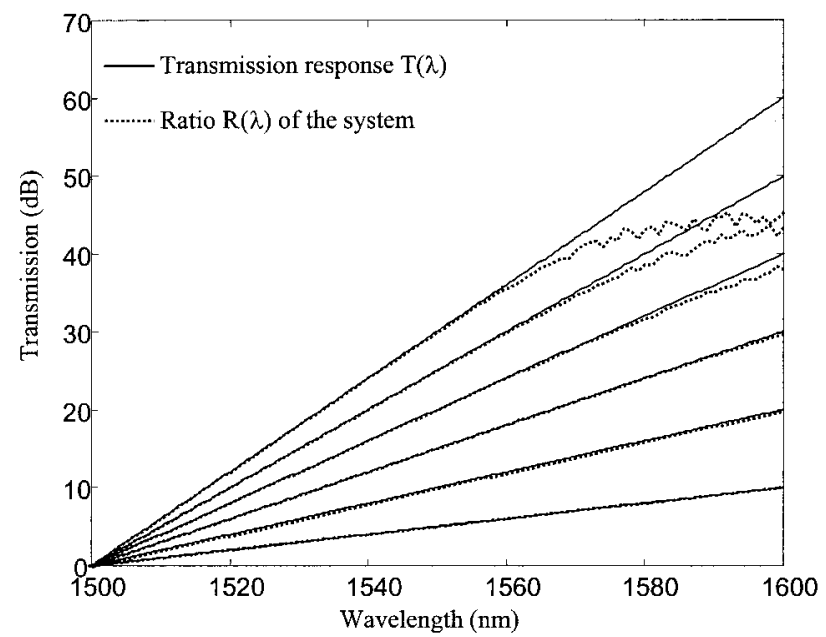

Fig. 3. Transmission responses of edge filters and corresponding ratios $R$ of systems.

i.e., $10 \log _{10}\left\{\exp \left[-4 \ln 2\left(\Omega^{2} / \Delta \lambda_{0}^{2}\right)\right]\right\}=-S$. Assume the transmission response of the edge filter in arm A is $T_{f}(\lambda)\left\{\right.$ denote $\bar{T}_{f}(f)=-10 \log _{10}\left[T_{f}(\lambda)\right]$ in decibels $\}$. It is known that photodiodes give the integral power over a wavelength range. Therefore the ratio of the outputs from the two photodiodes at a wavelength $\lambda_{0}$ is

$$
R\left(\lambda_{0}\right)=-10 \log _{10}\left[\frac{\left.\int I_{\lambda_{0}}(\lambda) T_{f}(\lambda) \mathrm{d} \lambda\right]}{\int I_{\lambda_{0}}(\lambda) \mathrm{d} \lambda}\right] .
$$

From this equation one knows that, for an ideal source, i.e., the SNR is infinite, the ratio $R\left(\lambda_{0}\right)$ is extremely close to the transmission response $\bar{T}_{f}\left(\lambda_{0}\right)$.

A simple case for the transmission response $\bar{T}_{f}(\lambda)$ of an edge filter in wavelength range $\left(\lambda_{1}, \lambda_{2}\right)$ is a linear function, i.e.,

$$
\bar{T}_{f}(\lambda)=\bar{T}_{f}\left(\lambda_{1}\right)+\frac{\left[\bar{T}_{f}\left(\lambda_{2}\right)-\bar{T}_{f}\left(\lambda_{1}\right)\right]}{\left(\lambda_{2}-\lambda_{1}\right)}\left(\lambda-\lambda_{1}\right) .
$$

With this linear transmission response, numerical examples are given below that model performances of the ratiometric wavelength-measurement system. Assume the input signal has a 55-dB SNR in a wavelength range from 1500 to $1600 \mathrm{~nm}$ with a maximum $r=1 \mathrm{~dB}$. Six edge filters are considered, of which $\bar{T}_{f}\left(\lambda_{1}\right)=0 \mathrm{~dB}$ and $\bar{T}_{f}\left(\lambda_{2}\right)$ is chosen to be from 10 to $60 \mathrm{~dB}$ with an increment of $10 \mathrm{~dB}$. Corresponding numerical results of the output ratio $R$ are shown in Fig. 3. Transmission responses of these six edge filters are also presented in Fig. 3. From these numerical results one can see that, with the increase in slope of the transmission response of the edge filters for a given measurable wavelength range, the calcu- 


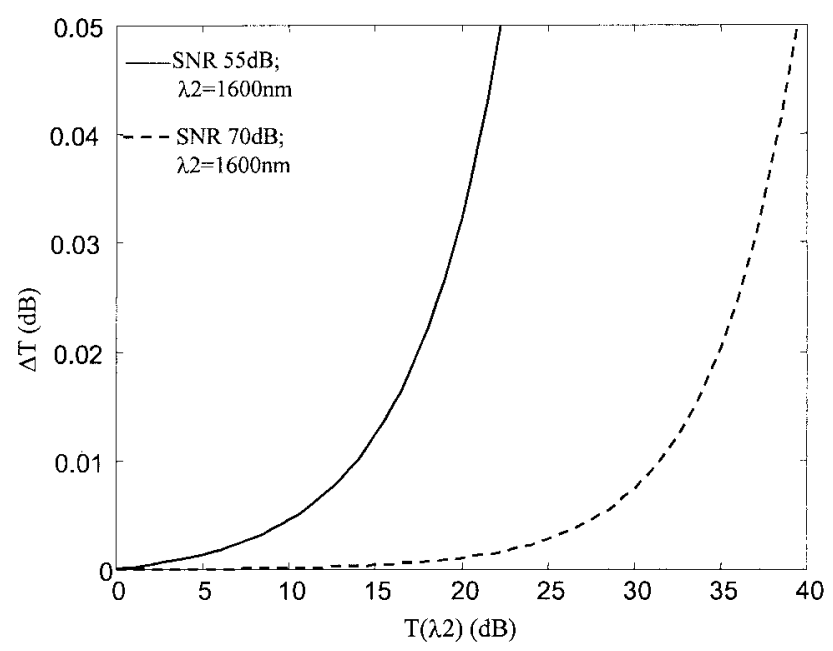

(a)

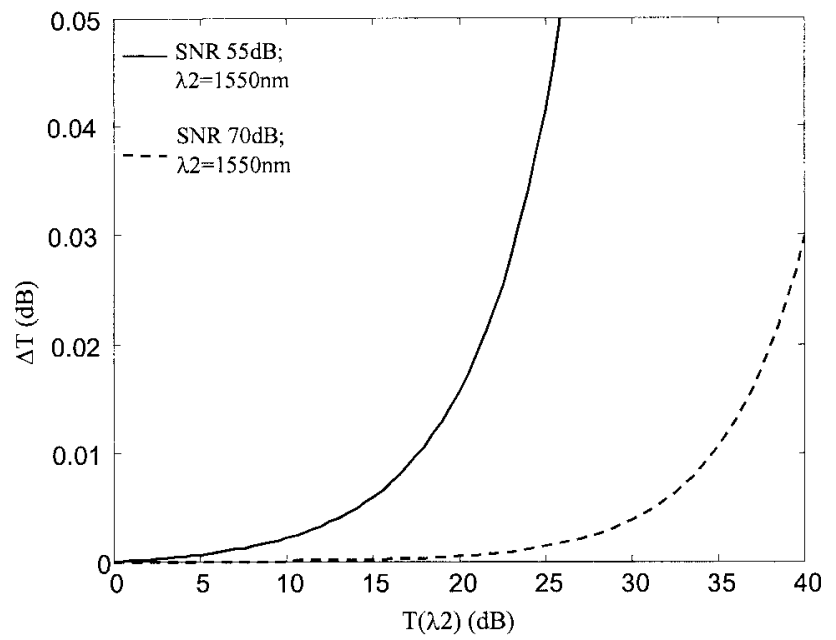

(b)

Fig. 4. Differences between transmission response and output ratio of the system at (a) $\lambda_{2}=1600 \mathrm{~nm}$ and (b) $\lambda_{2}=1550 \mathrm{~nm}$.

lated ratios $R$ start to diverge from the transmission response of the edge filters at the end region of the wavelength range as a result of the limited SNR of the source. This indicates that the straightforward design approach mentioned in the introduction could be wrong and there exists a maximal slope for the transmission response of the edge filter beyond which significant measurement error will occur because of the finite SNR of the signal source.

To find the maximal transmission response slope for a given wavelength range one can use the difference between transmission response and the output ratio $R$ of the system at wavelength $\lambda_{2}$, i.e., $\Delta T$ $=\bar{T}\left(\lambda_{2}\right)-R\left(\lambda_{2}\right)$. Figures $4(\mathrm{a})$ and $4(\mathrm{~b})$ give the calculated $\Delta T$ against transmission $\bar{T}\left(\lambda_{2}\right)$ for two source signals with SNRs of 55 and $70 \mathrm{~dB}$, respectively. The considered wavelength range in Figs. 4(a) and 4(b) is (in nanometers) $(1500,1600)$ and $(1500,1550)$, respectively. Assume that the difference is required to be within $0.01 \mathrm{~dB}$ (comparable with the precision of the power detectors). From these calculation results, one can see that for the signal with a SNR of $55 \mathrm{~dB}$ and measurement range of $(1500 \mathrm{~nm}, 1600 \mathrm{~nm})$, the transmission at $1600 \mathrm{~nm}$ should be no larger than $13 \mathrm{~dB}$ (i.e., slope $0.13 \mathrm{~dB} / \mathrm{nm}$ ). When the required measurable wavelength range is $(1500 \mathrm{~nm}$, $1550 \mathrm{~nm}$ ), the transmission at $1550 \mathrm{~nm}$ should be no larger than $17 \mathrm{~dB}$ (i.e., slope $0.34 \mathrm{~dB} / \mathrm{nm}$ ). When the SNR of the input signal is higher at $\sim 70 \mathrm{~dB}$, the transmission at $1600 \mathrm{~nm}$ should be no larger than $32 \mathrm{~dB}$ (i.e., slope $0.32 \mathrm{~dB} / \mathrm{nm}$ ) and the transmission at $1550 \mathrm{~nm}$ should be no larger than $34 \mathrm{~dB}$ (i.e., slope $0.68 \mathrm{~dB} / \mathrm{nm}$ ). In the context of the limited precision of the power detectors, one can see that the SNR of the signal source has a significant impact on the transmission spectrum of edge filters and, as a consequence, affects the measurement system's resolution.

\section{Experimental Investigation of Impact of the Signal-to-Noise Ratio}

To verify the above theoretical modeling, a ratiometric wavelength-measurement system was built and corresponding experimental measurements were carried out. A tunable laser is chosen as the signal source, and its corresponding SNR is $>40 \mathrm{~dB}$. Eight edge filters operating at a wavelength range $(1500 \mathrm{~nm}, 1600 \mathrm{~nm})$ with different spectral responses were used, which utilize the wavelength sensitivity of bend loss for a single-mode fiber. ${ }^{10}$ It should be noted that the transmission responses of the edge filters are not strictly linear functions of the wavelength as used in the theoretical modeling. Figure 5(a) gives the measured transmission responses of the edge filters by an optical spectral analyzer (circles). Simulated output ratios $R$ of the system with the above model are also presented as dotted curves. Figure 5(b) gives corresponding measured ratios $R$ of the system with a dual-channel power meter. Comparing Figs. 5(a) and $5(\mathrm{~b})$, one can see that the simulated results have a good agreement with the measured results. Again the experimental results show that, because of the limited SNR of the source, with the increase in the slope of the transmission response of the edge filter, the output ratios $R$ diverge from the actual transmission response of the edge filters within the end region of the wavelength range, and furthermore these differences vary from each measurement, which means that it is not suitable for a wavelength-measurement application. Therefore the slope of the transmission response of edge filters is strictly limited. To find the optimal transmission response for a required wavelength range or find the measurable wavelength range for a given transmission response of edge filter, one can apply the theoretical model and design method demonstrated in Section 2. For the SNR of the source used in the experiment, both the theoretical model and the experiment show that the measurable wavelength range for the uppermost transmission response is approximately $(1500 \mathrm{~nm}$, $1550 \mathrm{~nm}$ ) and the corresponding slope is $\sim 0.3 \mathrm{~dB} / \mathrm{nm}$. 


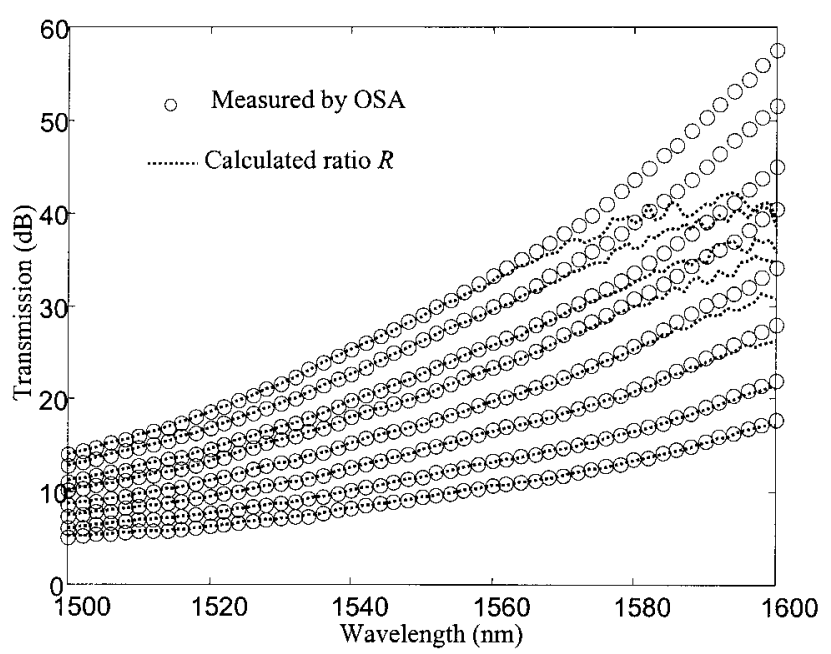

(a)

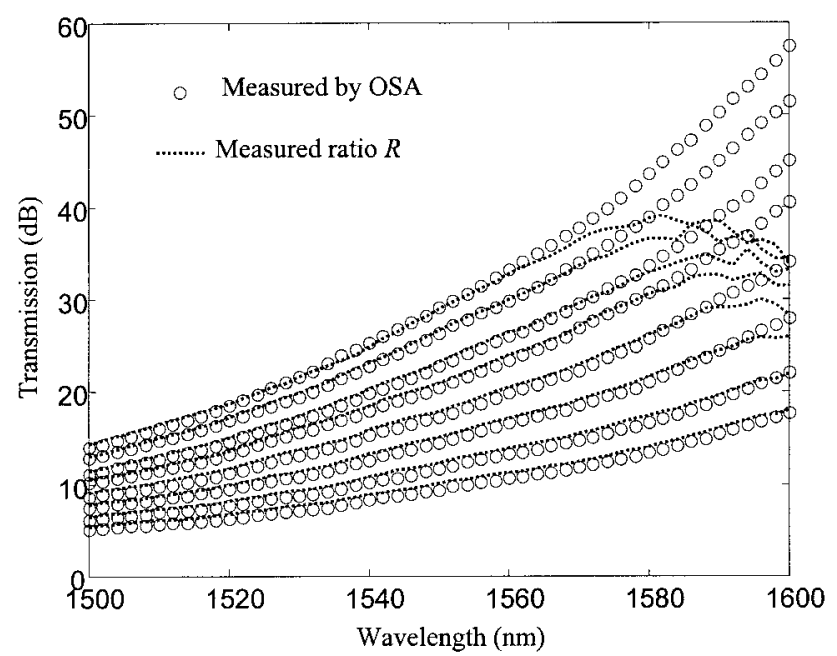

(b)

Fig. 5. (a) Measured transmission responses of the edge filters and calculated ratios of the system, (b) measured transmission responses of the edge filters and measured ratios of the system.

\section{Conclusion}

The spectral responses of edge filters employed in wavelength-measurement systems have been studied theoretically and experimentally. A simple model has been presented that describes a ratiometric wavelength-measurement system. The design of the transmission response of an edge filter has been demonstrated. The simulation results and experimental results have shown that the limited SNR of the signal source affects not only the maximum slope of the edge filter's spectral response over a given wavelength range, but also affects the wavelength range. According to the theoretical and experimental results presented in this paper, for a SNR of $40 \mathrm{~dB}$, the widest wavelength range is $\sim 50 \mathrm{~nm}$ with an edge filter's slope of $0.3 \mathrm{~dB} / \mathrm{nm}$. The widest wavelength range increases as the SNR of the source improves for a given slope of the edge filter or as the slope decreases for a given SNR of the source.

\section{References}

1. J. Mora, J. Luis Cruz, M. V. Andres, and R. Duchowicz, "Simple high-resolution wavelength monitor based on a fiber Bragg grating," Appl. Opt. 43, 744-749 (2004).

2. S. M. Melle, K. Liu, and R. M. Measures, "A passive wavelength demodulation system for guided-wave Bragg grating sensors," IEEE Photonics Technol. Lett. 4, 516-518 (1992).

3. M. A. Davis and A. D. Kersey, "All fiber Bragg grating strainsensor demodulation technique using a wavelength division coupler," Electron. Lett. 30, 75-76 (1994).

4. A. B. L. Ribeiro, L. A. Ferreira, M. Tsvekov, and J. L. Santos, "All-fiber interrogation technique for fiber Bragg sensors using a biconical fiber filter," Electron. Lett. 32, 382-383 (1996).

5. Y. Liu, L. Zhang, and I. Bennion, "Fabricating fibre edge filters with arbitrary spectral response based on titled chirped grating structures," Meas. Sci. Technol. 10, L1-L3 (1999).

6. B. Mason, S. P. Denbarrs, and L. A. Coldren, "Tunable sampled-grating DBR lasers with integrated wavelength monitors," IEEE Photonics Technol. Lett. 10, 1085-1087 (1998).

7. J. J. Lepley and A. S. Siddiqui, "Primary referenced DWDM frequency comb generator," IEE Proc. Optoelectron. 146, 121124 (1999).

8. M. Bass, E. W. Van Stryland, D. R. William, and W. L. Wolfe, Handbook of Optics, 2nd ed. (McGraw-Hill, 1995), Vol. 1, Part 4.

9. M. G. Xu, H. Geiger, and J. P. Dakin, "Modeling and performance analysis of a fiber Bragg grating interrogation system using an acousto-optic tunable filter," J. Lightwave Technol. 14, 391-396 (1996).

10. Q. Wang, G. Farrell, and T. Freir, "Theoretical and experimental investigations of macrobend losses for standard single mode fibers," Opt. Exp. 13, 4476-4484 (2005). 